\title{
Natural Operators Lifting 1-Forms to Bundles of Weil Contact Elements
}

\author{
MIROSLAV KUREŠ AND WŁODZIMIERZ M. MIKULSKI
}

\begin{abstract}
All natural operators lifting 1-forms from $m$ dimensional manifolds to the bundle functor $K^{A}$ of Weil contact elements are classified for the case of dwindlable Weil algebras. New results concerning subalgebras of fixed elements and the relation to dwindlable Weil algebras are stated. For some monomial Weil algebras, the Weil contact elements are interpreted geometrically.
\end{abstract}

\section{INTRODUCTION}

The modern development of differential geometry clarified that differential geometric objects form fiber bundles over manifolds. These bundles are studied in the form of so called natural bundles. A natural bundle over $m$-dimensional manifolds is a covariant functor $F$ from the category of $m$-dimensional manifolds and local diffeomorphisms satisfying the following conditions:

B I. (Prolongation) For every $m$-dimensional manifold $M, F M$ is a fibered manifold over $M$. Then $\pi_{M}: F M \rightarrow M$ is the induced projection and for a point $x \in M$ we denote $F_{x} M=\pi_{M}^{-1}(x)$ the fiber over $x$.

B II. (Locality) For every inclusion $i: U \rightarrow M$ of an open submanifold $U$ is $F U=\pi_{M}^{-1}(U)$ and $F i: F U \rightarrow F M$ is the inclusion of $\pi_{M}^{-1}(U)$ into $F M$.

2000 Mathematics Subject Classification. 58A32, 58A20, 12D05, 53A55, $58 \mathrm{H} 99$.

Key words and phrases. Weil algebra, contact element, lifting.

The first author was supported by GA CR, grant No. 201/99/0296.

This paper is in final form and no version of it will be submitted for publication elsewhere. 
B III. (Regularity) For every smoothly parameterized family $\varphi_{t}: M \rightarrow N$ of local diffeomorphisms is $F \varphi_{t}: F M \rightarrow F N$ a smoothly parameterized family of fibered local isomorphisms.

Nevertheless, it was proved that prolongation and locality conditions imply the regularity condition, [6]. There are general important questions regarding the classification of geometric constructions of given type. The lifting of geometric objects (as vector fields, forms, connections, etc.) to fiber bundles is one of the most important examples of such constructions. Let $F, G$ be natural bundles over $m$-dimensional manifolds, $m+n=\operatorname{dim} F \mathbb{R}^{m}$ and let $H$ be a natural bundle over $(m+n)$-dimensional manifolds. We denote $C^{\infty} G M$ and $C^{\infty} H F M$ the spaces of local sections of $G M \rightarrow M$ and $H F M \rightarrow$ $F M$, respectively. Elements of these spaces are called geometric $G$ and $H$-objects. A lifting to $F$ of geometric $G$-objects from $M$ to geometric $H$-objects on $F M$ is a family $\Lambda=\left\{\Lambda_{M}\right\}: G \rightsquigarrow H F$ of mappings

$$
\Lambda_{M}: C^{\infty} G M \rightarrow C^{\infty} H F M
$$

satisfying the following conditions:

L I. (Prolongation) If $s \in C^{\infty} G M$ is defined on an open subset $U \subset M$ then $\Lambda_{M}(s) \in C^{\infty} H F M$ is defined on $F U \subset F M$.

L II. (Naturality) For every diffeomorphism $\varphi: M \rightarrow N$, if objects $s_{1} \in C^{\infty} G M, s_{2} \in C^{\infty} G N$ are $\varphi$-related, then $\Lambda_{M}\left(s_{1}\right) \in C^{\infty} H F M, \Lambda_{N}\left(s_{2}\right) \in C^{\infty} H F N$ are $F \varphi$-related (i.e. $\left.s_{2} \circ \varphi=G \varphi \circ s_{1} \Rightarrow \Lambda_{N}\left(s_{2}\right) \circ F \varphi=H F \varphi \circ \Lambda_{M}\left(s_{1}\right)\right)$.

We say that a lifting $\Lambda=\left\{\Lambda_{M}\right\}$ to $F$ satisfies the regularity condition if

L III. (Regularity) If $s_{t} \in C^{\infty} G M$ is a smooth family of local fields of geometric objects on $M$, then $\Lambda_{M}\left(s_{t}\right) \in C^{\infty} H F M$ is also a smooth family of local fields of geometric objects on $F M$.

The prolongation and naturality conditions imply immediately

L IV. (Locality) If $s_{1}, s_{2} \in C^{\infty} G M$ are objects such that $s_{1 \mid U}=$ $s_{2 \mid U}$ for some open subset $U \subset M$, then $\Lambda_{M}\left(s_{1}\right)_{\mid F U}=$ $\Lambda_{M}\left(s_{2}\right) \mid F U$.

The regular lifting represents a so called natural operator. (We used the term of natural operator only in this sense here.) The theory of natural bundles and operators including methods for finding natural operators is very well presented in the monographical work [6]. 
Liftings are accentuated e.g. in [3], where an example of a lifting not satisfying the regularity (L III) is also given. In this paper, we study the problem of the lifting of a 1-form on a manifold $M$ into a 1form on the bundle $K^{A} M$ of Weil contact elements on $M$. In other words, we solve the problem how a 1-form on $M$ induces canonically a 1 -form on $K^{A} M$. Results of this problem are formulated in Section 3. We refer that the problem of the lifting of vector fields to bundles of Weil contact elements was solved quite recently in [9]. The properties of the bundle $K^{A} M$ depend substantially on the Weil algebra $A$. The lifting of vector fields and 1-forms initiates a round of questions in commutative algebra. Accordingly, we derive some necessary algebraic results in Section 1, which can be viewed independently. Section 2 is devoted to the geometrical interpretation of contact elements. We present their conceivable characterization for the case of some monomial algebras. All manifolds and maps in the paper are assumed to be of class $C^{\infty}$.

\section{Weil Algebras}

\subsection{The expression of a Weil algebra and their elements.}

The Weil algebra $A$ is a local commutative $\mathbb{R}$-algebra with identity, the nilpotent ideal $\mathfrak{n}$ of which has a finite dimension as a vector space and $A / \mathfrak{n}=\mathbb{R}$. We call the order of $A$ the minimum $\operatorname{ord}(A)$ of the integers $r$ satisfying $\mathfrak{n}^{r+1}=0$. (Some authors use the concept of Loevy length $L(A)$ in this description; but $L(A)$ is nothing else than $\operatorname{ord}(A)+1$.) Further, the integer $\mathrm{w}(A)=\operatorname{dim}\left(\mathfrak{n} / \mathfrak{n}^{2}\right)$ is called the width of $A$. One can assume that the Weil algebra is a finite dimensional factor $\mathbb{R}$-algebra of the algebra $\mathbb{R}\left[t^{1}, \ldots, t^{k}\right]$ of real polynomials in indeterminates $t^{1}, \ldots, t^{k}$, where $k=\mathrm{w}(A)$. It means, the Weil algebra $A$ has the form

$$
\mathbb{R}\left[t^{1}, \ldots, t^{k}\right] / \mathfrak{i}
$$

where $\mathfrak{m}^{r+1} \subset \mathfrak{i} \subset \mathfrak{m}^{2}$ for some $r, \mathfrak{m}=\left\langle t^{1}, \ldots, t^{k}\right\rangle$ being the maximal ideal of $\mathbb{R}\left[t^{1}, \ldots, t^{k}\right]$. Then $\operatorname{ord}(A)=r$. Moreover, $\mathfrak{i}$ is called the adequate ideal for $A$ in $\mathbb{R}\left[t^{1}, \ldots, t^{k}\right]$ and it has a finite set of generators as the algebra $\mathbb{R}\left[t^{1}, \ldots, t^{k}\right]$ is noetherian.

For a Weil algebra $A$, we introduce two new characteristic integers now. For a Weil algebra $A$ in the form (1), we denote by $\mathcal{G}$ the set of generators in $\mathfrak{i}$. As we say that the length of a polynomial $P$ is the minimal number of monomials the sum of which $P$ is, we define 
$\mu(\mathfrak{i}, \mathcal{G})$ as the length of the longest generator of $\mathfrak{i}$ belonging to $\mathcal{G}$ and $\nu(\mathfrak{i}, \mathcal{G})$ as the number of generators of $\mathfrak{i}$ belonging to $\mathcal{G}$ having the length $\mu(\mathfrak{i}, \mathcal{G})$. Further, we define $\mu(A)$ as the minimum of $\mu(\mathfrak{i}, \mathcal{G})$ with respect to all expressions of $A$ in the form (1) and all possible sets of generators of $\mathfrak{i}$. So, we can assume that the expression of $A$ satisfies (1) and

$$
\mu(\mathfrak{i}, \mathcal{G})=\mu(A)
$$

and we define $\nu(A)$ as the minimum of $\nu(\mathfrak{i}, \mathcal{G})$ with respect to all expressions of $A$ in the form (1) and (2) and all possible sets of generators of $\mathfrak{i}$. Thus, we can assume that the expression of $A$ satisfies (1), (2) as well as

$$
\nu(\mathfrak{i}, \mathcal{G})=\nu(A)
$$

and we shall call it the elementary polynomial expression of $A$. The case $\mu(A)=1$ is usually named that $A$ is monomial. (Weil algebras of higher order nonholonomic, semiholonomic and holonomic functors of velocities playing a leading role in analytical mechanics are presented in [8]. All of them are monomial.) We shall need the following assertions:

Lemma 1. If $\mathrm{w}(A)=1$, then $\mu(A)=1$ and $\nu(A)=1$.

Proof. The lemma is trivial.

Lemma 2. If $\operatorname{ord}(A) \leq 2$ and $\mathrm{w}(A)=2$, then $\mu(A)=1$.

Proof. See [5], Lemma 3.8.

Lemma 3. Let $\mathbb{R}\left[t^{1}, \ldots, t^{k}\right] / \mathfrak{i}$ be an elementary polynomial expression of a Weil algebra $A$ with the relevant set of generators $\mathcal{G}$ and with $\mu(A)=2$. Let $Q_{1}, Q_{2}$ be monomials in $t^{1}, \ldots, t^{k}, 1 \leq \operatorname{deg}\left(Q_{1}\right)<$ $\operatorname{deg}\left(Q_{2}\right) \leq \operatorname{ord}(A)$ and let $l \in \mathbb{N}, l>\operatorname{ord}(A)-\operatorname{deg}\left(Q_{2}\right)$. If

$$
Q_{2}=\alpha t^{j_{1}} \ldots t^{j_{l}} Q_{1}
$$

for some $\alpha \in \mathbb{R}$ and some $j_{1}, \ldots, j_{l}$ from $\{1, \ldots, k\}$ is satisfied, then $Q_{1}+Q_{2} \notin \mathcal{G}$.

Proof. One can easily deduce that $Q_{2} \in \mathfrak{i}$.

Let $A=\mathbb{R}\left[t^{1}, \ldots, t^{k}\right] / \mathfrak{i}$ be a monomial Weil algebra, i.e. $\mu(A)=1$. It is well-known, that $\mathbb{D}_{k}^{r}$ can be viewed as the algebra of truncated polynomials. There is a canonical epimorphism $p_{A}: \mathbb{D}_{k}^{r} \rightarrow A$ : if a monomial $Q, \operatorname{deg}(Q) \leq r$, belongs to generators of $\mathfrak{i}$, then it suffices 
add the new truncating condition $Q=0$ to the polynomial expression of elements. (The general construction of algebra epimorphisms $\mathbb{D}_{k}^{r} \rightarrow A$ is described in [1].) Then one can express elements of monomial $A$ as polynomials in $t^{1}, \ldots, t^{k}$ in the canonical way and $A$ can be viewed as an algebra of "unevenly truncated" polynomials. We shall denote these polynomials by

$$
P[A]\left(t^{1}, \ldots, t^{k}\right) .
$$

The following lemma represents an easily obtained result regarding the induced automorphism.

Lemma 4. Let $A=\mathbb{R}\left[t^{1}, \ldots, t^{k}\right] / \mathfrak{i}$ be a monomial Weil algebra, $\mathfrak{m}^{r+1} \subset \mathfrak{i} \subset \mathfrak{m}^{r}$, and let the linear part of $F \in A$ ut $\mathbb{D}_{k}^{r}$ be the identity. Then $F$ induces $\bar{F} \in$ Aut $A$ through the canonical epimorphism $p_{A}$.

Proof. Evidently, $F$ preserves i.

1.2. The subalgebra of fixed elements. In [9], the bijection between all natural operators lifting vector fields from $m$-dimensional manifolds to bundles of Weil contact elements and the subalgebra of fixed elements $S A=\{a \in A ; \phi(a)=a$ for all $\phi \in$ Aut $A\}$ of a Weil algebra $A$ is determined. Although in the absolute most of geometrically motivated illustrations is $S A=\mathbb{R} \cdot 1$, there are Weil algebras the subalgebra of fixed elements of which is nontrivial, i.e. $S A \supsetneqq \mathbb{R} \cdot 1$. Two types of sufficient conditions for the triviality of $S A$ are described in [9], Proposition 1 and Proposition 2. The example of $A$ with nontrivial $S A$ is e.g. $A=\mathbb{R}[s, t] /\left\langle s t^{2}+s^{4}, s^{2} t+t^{5}\right\rangle+\mathfrak{m}^{6}$. In view of the considerable importance of this problem we state the following new results.

Proposition 1. If $\operatorname{ord}(A) \leq 3$ and $\mathrm{w}(A)=2$, then $S A=\mathbb{R} \cdot 1$.

Proof. First, it is clear that for $\mu(A)=1$ there is an expression of $A=\mathbb{R}\left[t^{1}, \ldots, t^{k}\right] / \mathfrak{i}$, where $\mathfrak{i}$ is a homogeneous ideal and that is why $S A=\mathbb{R} \cdot 1$ as to [9], Proposition 1 . Lemma 2 says that for $\operatorname{ord}(A) \leq 2$ and $\mathrm{w}(A)=2$ is $\mu(A)=1$ and we have actually proved the assertion for $\operatorname{ord}(A) \leq 2$. We suppose $\operatorname{ord}(A)=3$ now. It follows from the proof of Proposition 3.9 in [5], that either

$$
\begin{aligned}
& \bar{s}=\tau s \\
& \bar{t}=\tau t
\end{aligned}
$$


$(\tau \neq 0)$ belongs to Aut $A$ (eventually, after an application of a change of variables) or $\mathfrak{i}$ can be generated by monomials and just one binomial of the form $Q_{1}+Q_{2}$, where $Q_{1}=Q_{1}(s, t), Q_{2}=Q_{2}(s, t)$ are monomials of degrees 2 and 3 , respectively. The equation

$$
Q_{1}\left(\tau_{1}, \tau_{2}\right)=Q_{2}\left(\tau_{1}, \tau_{2}\right)
$$

has a solution with $\tau_{1}>1$ and $\tau_{2}>1$ in all cases (see Lemma 3 ). Then

$$
\begin{aligned}
& \bar{s}=\tau_{1} s \\
& \bar{t}=\tau_{2} t
\end{aligned}
$$

belongs to Aut $A$ and preconditions of Proposition 2 from [9] are satisfied. Hence $S A=\mathbb{R} \cdot 1$.

We see that the mentioned Weil algebra with the nontrivial subalgebra of fixed elements has the order 5 and the width 2 . We have also proved that there is no such an algebra for $\operatorname{ord}(A) \leq 3$ and $\mathrm{w}(A)=2$. We enhance our results about Weil algebras with the nontrivial subalgebra of fixed elements now.

Proposition 2. Let $A=\mathbb{R}\left[t^{1}, \ldots, t^{k}\right] / \mathfrak{i}$ be a Weil algebra with the order $r$, the width $k, \mathfrak{m}^{r+1} \subset \mathfrak{i} \subset \mathfrak{m}^{3}$ and with the nontrivial subalgebra of fixed elements. Then there is a Weil algebra $B$ with the nontrivial subalgebra of fixed elements, $\operatorname{ord}(B)=r, \mathrm{w}(B)=k+1$. The assumptions can be satisfied for $r=4$ and $k=2$.

Proof. Let the assumptions for $A$ are satisfied. We take the Weil algebra $B:=\mathbb{R}\left[s, t^{1}, \ldots, t^{k}\right] / \mathfrak{i}+\left\langle s^{2}, s t^{1}, \ldots, s t^{k}\right\rangle$. Then $\operatorname{ord}(B)=r$, $\mathrm{w}(B)=k+1$. Let $a$ be an element of $A$ such that $0 \neq a \in S A \cap \mathfrak{n}$. We apply a canonical inclusion $i: A \rightarrow B$ and view $a$ also as an element of $B$. Let $\phi \in$ Aut $B$ be an arbitrary automorphism, $a=\phi(b), b \in B$, $a \neq b$. Automorphisms are determined by the specifying of elements which map to $s, t^{1}, \ldots, t^{k}$. So, $\phi$ have a form

$$
\begin{aligned}
b^{0}+\alpha^{0} s & \longmapsto s \\
b^{1}+\alpha^{1} s & \longmapsto t^{1} \\
& \cdots \\
b^{k}+\alpha^{k} s & \longmapsto t^{k}
\end{aligned}
$$

where $\alpha^{0}, \ldots, \alpha^{k} \in \mathbb{R}$ and $b^{0}, \ldots, b^{k}$ are elements of $B$ which do not depend on $s$. As $\left(b^{0}+\alpha^{0} s\right)^{2}=\left(b^{0}\right)^{2}$ maps to $s^{2}$, then $\left(b^{0}\right)^{2}=0$ and 
therefore $b^{0}$ is not linear in any $t^{j}$, because $\mathfrak{i} \subset \mathfrak{m}^{3}$. That is why

$$
\begin{array}{lll}
b^{1} & \longmapsto t^{1} \\
& \cdots & \\
b^{k} & \longmapsto t^{k}
\end{array}
$$

is the expression of an automorphism of Aut $A$ (we view $b^{1}, \ldots, b^{k}$ as elements of $A$, too). As we suppose $a=\phi(b)$ and $a \neq b$, then some $\alpha^{j} s(j \in\{1, \ldots, k\})$ must make that $a$ and $b$ are different. But it means that $a$ contains $\tau t^{j}, 0 \neq \tau \in \mathbb{R}$, because $\alpha^{j} s$ vanishes in the second order. Let

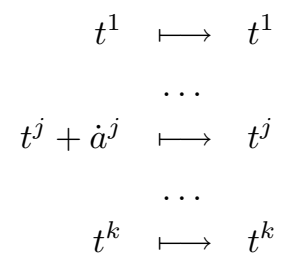

represent an automorphism of Aut $A$, in which $0 \neq \dot{a}^{j} \in A$ vanishes after multiplication by an arbitrary nilpotent element. (Such automorphisms always exist, because $\mathfrak{i} \subset \mathfrak{m}^{2}$.) This automorphism preserves $a$ as $a \in S A$. It implies $\tau \dot{a}^{j}=0$, i.e. $\tau=0$. It is contradiction; then $a=b$. It remains to give an example of a Weil algebra with the order $r$, the width $k$ and with the nontrivial subalgebra of fixed elements. Let $A=\mathbb{R}[s, t] /\left\langle s^{2} t+t^{4}, s^{3}+s t^{2}\right\rangle+\mathfrak{m}^{5}$. The elements of $A$ have a form

$$
k_{1}+k_{2} s+k_{3} t+k_{4} s^{2}+k_{5} s t+k_{6} t^{2}+k_{7} s^{3}+k_{8} s^{2} t+k_{9} t^{3}
$$

with the simultaneous vanishing of all monomials of the fifth or higher order in common with $s^{4}, s^{3} t, s^{2} t^{2}, s t^{3}, s^{2} t+t^{4}$ and $s^{3}+s t^{2}$. We shall describe automorphisms of $A$. The starting point for their specification is a form

$$
\begin{aligned}
& \bar{s}=A s+B t+C s^{2}+D s t+E t^{2}+F s^{3}+G s^{2} t+H t^{3} \\
& \bar{t}=I s+J t+K s^{2}+L s t+M t^{2}+N s^{3}+O s^{2} t+P t^{3} .
\end{aligned}
$$

The matrix $\left(\begin{array}{cc}A & B \\ I\end{array}\right)$ must be regular and we settle the conditions $\bar{s}^{4}=$ $0, \bar{s}^{3} \bar{t}=0, \bar{s}^{2} \bar{t}^{2}=0, \bar{s} \bar{t}^{3}=0, \bar{s}^{2} \bar{t}+\bar{t}^{4}=0$ and $\bar{s}^{3}+\bar{s}^{2}=0$ now. The condition $\bar{s}^{4}=0$ gives $B=0$. The conditions $\bar{s}^{3} \bar{t}=0, \bar{s}^{2} \bar{t}^{2}=0$, $\bar{s} \bar{t}^{3}=0$ give no new nontrivial relation. The condition $\bar{s}^{2} \bar{t}+\bar{t}^{4}=0$ gives $I=0, A^{2}=J^{3}$. The condition $\bar{s}^{3}+\bar{s} \bar{t}^{2}=0$ gives $E=0$, 
$A^{2}=J^{2}$. So, we obtain $J=1$ and $A=-1$ or $A=1$. Hence the automorphisms have the following form

$$
\begin{aligned}
& \bar{s}=\epsilon s+C s^{2}+D s t+F s^{3}+G s^{2} t+H t^{3} \\
& \bar{t}=t+K s^{2}+L s t+M t^{2}+N s^{3}+O s^{2} t+P t^{3},
\end{aligned}
$$

where $\epsilon \in\{-1,1\}$. Finally, we solve the equation

$$
\begin{aligned}
& k_{1}+k_{2} \bar{s}+k_{3} \bar{t}+k_{4} \bar{s}^{2}+k_{5} \bar{s} \bar{t}+k_{6} \bar{t}^{2}+k_{7} \bar{s}^{3}+k_{8} \bar{s}^{2} \bar{t}+k_{9} \bar{t}^{3} \\
& k_{1}+k_{2} s+k_{3} t+k_{4} s^{2}+k_{5} s t+k_{6} t^{2}+k_{7} s^{3}+k_{8} s^{2} t+k_{9} t^{3}
\end{aligned}
$$

for $k_{i}, i=1, \ldots, 9$, by use the described automorphisms. By comparing of coefficients standing at powers of $s$ and $t$, we find that $k_{2}=k_{3}=k_{4}=k_{5}=k_{6}=k_{7}=k_{9}=0$ and $k_{1}, k_{8}$ are arbitrary real coefficients. This means $S A=\left\{k_{1}+k_{8} s^{2} t\right\} \supsetneqq \mathbb{R} \cdot 1$. The assumption $\mathfrak{m}^{5} \subset \mathfrak{i} \subset \mathfrak{m}^{3}$ is also satisfied.

REMARK 1. Our nomination of the Weil algebra with $\operatorname{ord}(A)=4$ and $\mathrm{w}(A)=2$ is on the base of [5], Theorem 3.11, where local algebras with the unipotent identity component of the group of automorphisms are classified.

REMARK 2. In a similar way, we can prove (under assumptions of Proposition 2) that there is a Weil algebra $B$ with the nontrivial subalgebra of fixed elements, $\operatorname{ord}(B)=r, \mathrm{w}(B)=k+h$. Indeed, it suffices to take

$$
\begin{array}{r}
B:=\mathbb{R}\left[s^{1}, \ldots, s^{h}, t^{1}, \ldots, t^{k}\right] / \mathfrak{i}+\left\langle s^{1} s^{1}, \ldots, s^{1} s^{h}, \ldots, s^{h} s^{1}, \ldots,\right. \\
\left.s^{h} s^{h}, s^{1} t^{1}, \ldots, s^{1} t^{k}, \ldots, s^{h} t^{1}, \ldots, s^{h} t^{k}\right\rangle
\end{array}
$$

and to modify the proof.

1.3. Dwindlable Weil algebras. Of course, $\mathbb{R}$ is contained in every $\mathbb{R}$-algebra. Thus, the canonical algebra homomorphism $\kappa_{A}: A \rightarrow$ $\mathbb{R}$, where $A$ is a Weil algebra, can be viewed as the endomorphism $\kappa_{A}: A \rightarrow A$. We say that $A$ is dwindlable if there is an infinite sequence $\left\{\phi_{n}\right\}_{n=1}^{\infty}$ of automorphisms $\phi_{n} \in$ Aut $A$ such that $\phi_{n} \rightarrow \kappa_{A}$ for $n \rightarrow \infty$.

If for all $\tau \in \mathbb{R}, \tau \neq 0$, the automorphism $H_{\tau}: \mathbb{R}\left[t^{1}, \ldots, t^{k}\right] \rightarrow$ $\mathbb{R}\left[t^{1}, \ldots, t^{k}\right], H_{\tau}: P\left(t^{1}, \ldots, t^{k}\right) \mapsto P\left(\tau t^{1}, \ldots, \tau t^{k}\right)$, induces the automorphism $\bar{H}_{\tau}: \mathbb{R}\left[t^{1}, \ldots, t^{k}\right] / \mathfrak{i} \rightarrow \mathbb{R}\left[t^{1}, \ldots, t^{k}\right] / \mathfrak{i}$, (it arrives for all $A=\mathbb{R}\left[t^{1}, \ldots, t^{k}\right] / \mathfrak{i}$, where $\mathfrak{i}$ is a homogeneous ideal) then $A$ is dwindlable, as we come to $\kappa_{A}$ for $\tau \rightarrow 0$ or, more precisely, we take the 
sequence $\left\{\phi_{n}\right\}_{n=1}^{\infty}$ and e.g. $\frac{1}{n}$ in place of $\tau$. Admittedly, $A$ is dwindlable in other cases, too. For example, $A=\mathbb{R}[s, t] /\left\langle s^{2}+t^{3}\right\rangle+\mathfrak{m}^{4}$ does not dispose of the mentioned automorphism, but e.g. by the automorphism

$$
\begin{aligned}
& \bar{s}=\tau^{3} s \\
& \bar{t}=\tau^{2} t
\end{aligned}
$$

$(\tau \neq 0)$, for which we arrive at $\kappa_{A}$ for $\tau \rightarrow 0$. Actually, we have:

Proposition 3. If $\operatorname{ord}(A)=3, \mathrm{w}(A)=2, \mu(A)=2$ and $\nu(A)=1$, then $A$ is dwindlable.

Proof. $A=\mathbb{R}\left[t^{1}, \ldots, t^{k}\right] / \mathfrak{i}$ and there is the unique binomial among the generators of $i$. We can assume that this binomial is nonhomogeneous. Then we can state the full list of such Weil algebras and specify automorphisms which enable to obtain $\kappa_{A}$ in the limit. We have

(i) $A=\mathbb{R}[s, t] /\left\langle s^{2}+\alpha t^{3}\right\rangle+\mathfrak{m}^{4}$

(ii) $A=\mathbb{R}[s, t] /\left\langle s^{2}+\alpha t^{3}, s t\right\rangle+\mathfrak{m}^{4}$

(iii) $A=\mathbb{R}[s, t] /\left\langle s^{2}+\alpha t^{3}, s t^{2}\right\rangle+\mathfrak{m}^{4}$

(iv) $A=\mathbb{R}[s, t] /\left\langle s^{2}+\alpha s t^{2}\right\rangle+\mathfrak{m}^{4}$

(v) $A=\mathbb{R}[s, t] /\left\langle s^{2}+\alpha s t^{2}, t^{3}\right\rangle+\mathfrak{m}^{4}$

(vi) $A=\mathbb{R}[s, t] /\left\langle s t+\alpha t^{3}\right\rangle+\mathfrak{m}^{4}$

(vii) $A=\mathbb{R}[s, t] /\left\langle s t+\alpha t^{3}, s^{2}\right\rangle+\mathfrak{m}^{4}$

(viii) $A=\mathbb{R}[s, t] /\left\langle s t+\alpha t^{3}, s^{3}\right\rangle+\mathfrak{m}^{4}$

and automorphisms

$$
\begin{aligned}
& \bar{s}=\tau^{3} s \\
& \bar{t}=\tau^{2} t
\end{aligned}
$$

in the cases (i), (ii), (iii) and automorphisms

$$
\begin{aligned}
& \bar{s}=\tau^{2} s \\
& \bar{t}=\tau t
\end{aligned}
$$

in the cases (iv), (v), (vi) and (vii) ( $\tau \neq 0$ all the time).

Furthermore, we have the following assertion:

Proposition 4. If $A$ is a dwindlable Weil algebra, then its subalgebra $S A$ of fixed elements is trivial. Apart from that, there are non-dwindlable Weil algebras with trivial $S A$. 
Proof. First, we assume that $A$ is dwindlable and $S A \supsetneqq \mathbb{R} \cdot 1$. Then there is an element $a$ of $A$ such that $0 \neq a \in S A \cap \mathfrak{n}$. There is also an infinite sequence $\left\{\phi_{n}\right\}_{n=1}^{\infty}, \phi_{n} \in \operatorname{Aut} A, \phi_{n} \rightarrow \kappa_{A}$ for $n \rightarrow \infty$. It implies $0 \neq a=\phi_{n}(a) \rightarrow \kappa_{A}(a)=0$ and it is the contradiction. Now, we present an example of a non-dwindlable Weil algebra with trivial $S A$. Let $A=\mathbb{R}[s, t] /\left\langle s t^{2}+s^{5}, s^{2} t+t^{5}\right\rangle+\mathfrak{m}^{6}$. The elements of $A$ have a form

$$
\begin{aligned}
k_{1}+k_{2} s+k_{3} t+k_{4} s^{2}+ & k_{5} s t+k_{6} t^{2}+k_{7} s^{3} \\
& +k_{8} s^{2} t+k_{9} s t^{2}+k_{10} t^{3}+k_{11} s^{4}+k_{12} t^{4}
\end{aligned}
$$

with the simultaneous vanishing of all monomials of the sixth or higher order in common with $s^{2} t^{2}, s t^{3}, s^{3} t, s t^{2}+s^{5}$ and $s^{2} t+t^{5}$. We shall describe automorphisms of $A$. The starting point for their specification is a form

$$
\begin{array}{r}
\bar{s}=A s+B t+C s^{2}+D s t+E t^{2}+F s^{3}+G s^{2} t \\
+H s t^{2}+I t^{3}+J s^{4}+K t^{4} \\
\bar{t}=L s+M t+N s^{2}+O s t+P t^{2}+Q s^{3}+R s^{2} t \\
+S s t^{2}+T t^{3}+U s^{4}+V t^{4} .
\end{array}
$$

The matrix $\left(\begin{array}{cc}A & B \\ L & M\end{array}\right)$ must be regular and we settle the conditions $\bar{s}^{2} \bar{t}^{2}=0, \bar{s} \bar{t}^{3}=0, \bar{s}^{3} \bar{t}=0, \bar{s} \bar{t}^{2}+\bar{s}^{5}=0$ and $\bar{s}^{2} \bar{t}+\bar{t}^{5}=0$ now. The condition $\bar{s}^{2} \bar{t}^{2}=0$ gives $A=M=0$ (Variant I) or $B=L=0$ (Variant II). The condition $\bar{s} \bar{t}^{3}=0$ gives $C=0$ in the Variant I and $E=0$ in the Variant II. The condition $\bar{s}^{3} \bar{t}=0$ gives $P=0$ in the Variant I and $N=0$ in the Variant II. The condition $\bar{s} \bar{t}^{2}+\bar{s}^{5}=0$ gives $L^{2}=B^{4}, F=0$ in the Variant I and $M^{2}=A^{4}, I=0$ in the Variant II. The condition $\bar{s}^{2} \bar{t}+\bar{t}^{5}=0$ gives $B^{2}=L^{4}, T=0$ in the Variant I and $A^{2}=E^{2}+M^{4}, Q=0$ in the Variant II. Finally, we obtain $A=C=F=M=P=T=0, B$ and $L$ equal 1 or -1 in the Variant I or $B=E=I=L=N=Q=0, A$ and $M$ equal 1 or -1 in the Variant II. Hence the automorphisms have the following form

$$
\begin{aligned}
& \bar{s}=\epsilon_{1} t+D s t+E t^{2}+G s^{2} t+H s t^{2}+I t^{3}+J s^{4}+K t^{4} \\
& \bar{t}=\epsilon_{2} s+N s^{2}+O s t+Q s^{3}+R s^{2} t+S s t^{2}+U s^{4}+V t^{4}
\end{aligned}
$$

or

$$
\begin{aligned}
& \bar{s}=\epsilon_{1} s+C s^{2}+D s t+F s^{3}+G s^{2} t+H s t^{2}+J s^{4}+K t^{4} \\
& \bar{t}=\epsilon_{2} t+O s t+P t^{2}+R s^{2} t+S s t^{2}+T t^{3}+U s^{4}+V t^{4}
\end{aligned}
$$


where $\epsilon_{1}, \epsilon_{2} \in\{-1,1\}$. Evidently, $A$ is not dwindlable.

Finally, we solve the equation

$$
\begin{aligned}
& k_{1}+k_{2} \bar{s}+k_{3} \bar{t}+k_{4} \bar{s}^{2}+k_{5} \bar{s} \bar{t}+k_{6} \bar{t}^{2}+k_{7} \bar{s}^{3}+k_{8} \bar{s}^{2} \bar{t} \\
& +k_{9} \bar{s} \bar{t}^{2}+k_{10} \bar{t}^{3}+k_{11} \bar{s}^{4}+k_{12} \bar{t}^{4}=k_{1}+k_{2} s+k_{3} t+k_{4} s^{2} \\
& \quad+k_{5} s t+k_{6} t^{2}+k_{7} s^{3}+k_{8} s^{2} t+k_{9} s t^{2}+k_{10} t^{3}+k_{11} s^{4}+k_{12} t^{4}
\end{aligned}
$$

for $k_{i}, i=1, \ldots, 12$, by use the described automorphisms. By comparing of coefficients standing at powers of $s$ and $t$, we find that $k_{2}=k_{3}=k_{4}=k_{5}=k_{6}=k_{7}=k_{8}=k_{9}=k_{10}=k_{11}=k_{12}=0$ and $k_{1}$ is an arbitrary real coefficient. Thus $S A=\mathbb{R} \cdot 1$.

\section{Contact Elements}

2.1. Bundles of Weil contact elements. Weil algebras can be also viewed as finite dimensional factor $\mathbb{R}$-algebras of the algebra of germs $\mathcal{E}_{k}=C_{0}^{\infty}\left(\mathbb{R}^{k}, \mathbb{R}\right)$. The fact that ideals in $\mathcal{E}_{k}$ can be generated by some polynomials induces the corresponding ideal $\underline{i}$ in $\mathcal{E}_{k}$ for every Weil ideal $\mathfrak{i}$ in $\mathbb{R}\left[t^{1}, \ldots, t^{k}\right]$. Let $A=\mathcal{E}_{k} / \underline{\mathfrak{i}}$ be a Weil algebra and $M$ a $m$-dimensional manifold. Two maps $g, h: \mathbb{R}^{k} \rightarrow M, g(0)=h(0)=x$ are said to be $A$-equivalent, if $\alpha \circ g-\alpha \circ h \in \mathfrak{i}$ for every germ $\alpha$ of a smooth function on $M$ at $x$. Such an equivalence class will be denoted by $j^{A} g$ and called an $A$-velocity on $M$. The point $x=g(0)$ is said to be the target of $j^{A} g$. Denote by $T^{A} M$ the set of all $A$ velocities on $M$. $T^{A} M$ is called the Weil bundle. Let $\operatorname{reg} T^{A} M \subset$ $T^{A} M$ be the open subbundle of so called regular $A$-velocities on $M$, i.e. if $A=\mathcal{E}_{k} / \underline{i}$, then $j^{A} g \in \operatorname{reg} T^{A} M \subset T^{A} M$ if and only if $g: \mathbb{R}^{k} \rightarrow M$ is of rank $k$ at 0 . The contact element of type $A$ or Weil contact element on $M$ determined by $X \in \operatorname{reg} T^{A} M$ is the equivalence class Aut $A_{M}(X):=\{\phi(X) ; \phi \in$ Aut $A\}$. We denote by $K^{A} M$ the set of all Weil contact element (of type $A$ ) on $M . K^{A} M$ represents a peremptory generalization of classical contact element bundles, it is called the bundle of Weil contact elements and it plays the central role in our paper.

We obtain the classical contact element bundles introduced by C. Ehresmann in [2] by the choice $A=\mathbb{D}_{k}^{r}=\mathbb{R}\left[t^{1}, \ldots, t^{k}\right] / \mathfrak{m}^{r+1}$ with the usual denotation $K_{k}^{r} M$ instead $K^{\mathbb{D}_{k}^{r}} M$. (For the geometry of classical contact elements see e.g. [4].) Alternatively, the space of all $k$-dimensional subspaces in a vector space $V$ is called the Grassmann manifold or the Grassmannian. If $V=T_{x} M$, then 
such a Grassmannian is represented just by $\left(K_{k}^{1} M\right)_{x}$ and that is why $K_{k}^{1} M=\bigcup_{x \in M}\left(K_{k}^{1} M\right)_{x}$ is called the bundle of $k$-dimensional Grassmannians. Accordingly, $K_{k}^{r} M$ is sometimes called the bundle of $k$-dimensional Grassmannians of order $r$.

2.2. The characterization of Weil contact elements for monomial Weil algebras. An $m$-dimensional manifold $M$ is locally identified with $\mathbb{R}^{m}$ with usual coordinates $x^{i}, i=1, \ldots, m$. By the algebraically parameterized subset in $\mathbb{R}^{m}$ we mean a set $N \subset \mathbb{R}^{m}$ possessed of a parameterization $x^{i}=P^{i}$, where $P^{i}$ are real polynomials in at most $m-1$ indeterminates (parameters). In general, $N$ disposes of infinitely many such parameterizations; we denote by $\underline{N}$ the couple $\left(N, P^{i}\right)$, i.e. $N$ with a fixed parameterization.

Let $A$ be a monomial Weil algebra, $m-1 \geq \mathrm{w}(A)=k$, with elements taken as polynomials (4). We define how elements of $\operatorname{reg} T^{A} \mathbb{R}^{m}$ induce a submanifold of mentioned type. We can express elements of $A=\mathbb{R}\left[t^{1}, \ldots, t^{k}\right] / \mathfrak{i}$ as polynomials in the form

$$
k_{0}+k_{1} \mathbf{t}_{1}+\cdots+k_{q} \mathbf{t}_{q},
$$

where $\mathbf{t}_{1}, \ldots, \mathbf{t}_{q}$ are different elementary monomials of degree at least 1 (i.e. $\mathbf{t}_{h}=t^{j_{1}} \ldots t^{j_{s}} \notin \mathfrak{i}, 1 \leq s \leq r, j_{1}, \ldots, j_{s} \in\{1, \ldots, k\}$ for every $h=1, \ldots, q)$ and $k_{0}, k_{1}, \ldots, k_{q} \in \mathbb{R}$. Let $g: \mathbb{R}^{k} \rightarrow \mathbb{R}$ be a smooth function. We find easily coefficients of the polynomial $P[A]$ corresponding with elements of $A$ and satisfying equations

$$
\begin{aligned}
g(0) & =P[A](0) \\
\frac{\partial^{\left|\mathbf{t}_{h}\right|} g}{\partial \mathbf{t}_{h}}(0) & =\frac{\partial^{\left|\mathbf{t}_{h}\right|} P[A]}{\partial \mathbf{t}_{h}}(0),
\end{aligned}
$$

where $h=1, \ldots, q, \partial \mathbf{t}_{h}$ means $\partial t^{j_{1}} \ldots \partial t^{j_{s}}$, and by $\left|\mathbf{t}_{h}\right|$ is denoted the degree of the monomial $\mathbf{t}_{h} . P[A]$ is uniquely determined and represents only a slight generalization of the Taylor polynomial. Now, if we view $A$ as $\mathcal{E}_{k} / \underline{i}$, then $A$-velocity $j^{A} g$ is determined by $g: \mathbb{R}^{k} \rightarrow \mathbb{R}^{m}, g=\left(g^{1}\left(t^{1}, \ldots, t^{k}\right), \ldots, g^{m}\left(t^{1}, \ldots, t^{k}\right)\right)$ and we can realize the described assigning of polynomial $P^{i}[A]$ to $g^{i}, i=1, \ldots, m$. The regularity means that we postulate with the rank $k$ at 0 for $g: \mathbb{R}^{k} \rightarrow \mathbb{R}^{m}$ and implies that the described algebraically parameterized subsets

$$
x^{i}=P^{i}[A]\left(t^{1}, \ldots, t^{k}\right) .
$$

are (locally) $k$-dimensional submanifolds in $\mathbb{R}^{m}$. We shall denote them by $N[A]$ and call the osculating A-paraboloids. As above, $N[A]$ 
represents a couple $\left(N[A], P^{i}[A]\right)$ and we shall denote by $\mathcal{S}_{m}[A]$ the set of all $N[A]$ and by $\underline{\mathcal{S}}_{m}[A]$ the set of all $\underline{N}[A]$ for a given $A$. By an algebraic subset in $\mathbb{R}^{m}$ one usually means the set of zeros of some subset of $\mathbb{R}\left[t^{1}, \ldots, t^{k}\right]$. We say that $N[A] \in \mathcal{S}_{k+1}[A]$ is an adequately algebraic submanifold if there is a polynomial $Z=Z\left(x^{1}, \ldots, x^{k+1}\right)$, $1 \leq \operatorname{deg}(Z) \leq \operatorname{ord}(A)$, for which $Z\left(P^{1}[A], \ldots, P^{k+1}[A]\right)=0$. We shall denote by $\mathcal{S}_{k+1}^{*}[A]$ the subset of all adequately algebraic submanifolds in $\mathcal{S}_{k+1}[A]$.

Proposition 5. Let $A$ be a monomial Weil algebra. There is a bijection between $\operatorname{reg} T^{A} \mathbb{R}^{m}$ and $\underline{\mathcal{S}}_{m}[A]$.

Proof. It follows from the described construction of elements of $\underline{\mathcal{S}}_{m}[A]$ that $\left.\alpha \circ g-\alpha \circ\left(P^{1}[A], \ldots, P^{m}[A]\right)\right) \in \mathfrak{i}$ for every germ $\alpha$ of a smooth function on $\mathbb{R}^{m}$ at $g(0)=x$. On the other hand, every $\left(P^{1}[A], \ldots, P^{m}[A]\right)$ must belong to any $A$-velocity.

REMARK 3. Nevertheless, elements of reg $T^{A} \mathbb{R}^{m}$ coalescing in $K^{A} \mathbb{R}^{m}$ give in general different elements of $\mathcal{S}_{m}[A]$, e.g. for $A=\mathbb{D}_{1}^{2}$ and $m=2, N_{1}[A]$ given by

$$
\begin{aligned}
& x=t \\
& y=t^{2}
\end{aligned}
$$

and $N_{2}[A]$ given by

$$
\begin{aligned}
& x=t+t^{2} \\
& y=t^{2}
\end{aligned}
$$

are different curves in $\mathbb{R}^{2}$ obtained by the application of the automorphism $t \mapsto t+t^{2}$ belonging to Aut $\mathbb{D}_{1}^{2}$. So, we can present nothing but a bijection between $K^{A} \mathbb{R}^{m}$ and $\underline{\mathcal{S}}_{m}[A] / \equiv_{A}$, where $\equiv_{A}$ is the equivalence relation on $\underline{\mathcal{S}}_{m}[A]$ defined as follows: $\underline{N}_{1}[A] \equiv_{A} \underline{N}_{2}[A]$ if and only if there exists a local diffeomorphism $\psi: \mathbb{R}^{m} \rightarrow \mathbb{R}^{m}$, $\psi(0)=0$, satisfying $\psi^{*} \underline{\mathfrak{i}}=\underline{\mathfrak{i}}$ and $\operatorname{germ}_{0}\left(P_{1}[A] \circ \psi-P_{2}[A]\right) \in \underline{\mathfrak{i}}$.

\subsection{Examples of osculating $A$-paraboloids.}

Example A. $A=\mathbb{D}_{k}^{1}$. If $k=1$, then elements of $A$ have the form $k_{0}+k_{1} t$ and induce submanifolds of $\mathbb{R}^{m}$ given by $x^{i}=\alpha^{i}+\beta^{i} t$, $\alpha^{i}, \beta^{i} \in \mathbb{R}$. Of course, these submanifolds are straight lines. The generalization for an arbitrary $k$ is easy and we obtain $k$-planes (which constitute the bundle of $k$-dimensional Grassmannians). Evidently, $\mathcal{S}_{k+1}^{*}\left[\mathbb{D}_{k}^{1}\right]=\mathcal{S}_{k+1}\left[\mathbb{D}_{k}^{1}\right]$. 
Example B. $A=\mathbb{D}_{1}^{2}$. Elements of $A$ have a form $k_{0}+k_{1} t+k_{2} t^{2}$ and for $m=k+1=2$ they induce curves

$$
\begin{aligned}
& x=\alpha+\beta t+\gamma t^{2} \\
& y=\delta+\zeta t+\eta t^{2},
\end{aligned}
$$

$\alpha, \beta, \gamma, \delta, \zeta, \eta \in \mathbb{R}, \operatorname{rank}\left(\begin{array}{c}\beta \\ \zeta\end{array}\right)=1$. However, in the suitable coordinate system it is possible assume $\alpha=\delta=0, \beta=1, \zeta=0$. We have

$$
\begin{aligned}
& x=t+\gamma t^{2} \\
& y=\eta t^{2},
\end{aligned}
$$

so it follows that

$$
x^{2}-\frac{2 \gamma}{\eta} x y+\frac{\gamma^{2}}{\eta^{2}} y^{2}-\frac{1}{\eta} y=0
$$

and by standard procedure we evaluate that it embodies the parabola with the normal equation

$$
\left(1+\frac{\gamma^{2}}{\eta^{2}}\right) x^{2}-\frac{1}{\sqrt{\gamma^{2}+\eta^{2}}} y=0 .
$$

Hence $\mathcal{S}_{2}^{*}\left[\mathbb{D}_{1}^{2}\right]=\mathcal{S}_{2}\left[\mathbb{D}_{1}^{2}\right]$ was proved.

Example C. $A=\mathbb{D} \otimes \mathbb{D}$. Elements of $A$ have a form $k_{0}+k_{1} s+k_{2} t+$ $k_{3} s t$ and for $m=k+1=3$ they induce surfaces

$$
\begin{aligned}
& x=\alpha+\beta s+\gamma t+\delta s t \\
& y=\zeta+\eta s+\theta t+\iota s t \\
& z=\kappa+\lambda s+\mu t+\nu s t,
\end{aligned}
$$

$\alpha, \beta, \gamma, \delta, \zeta, \eta, \theta, \iota, \kappa, \lambda, \mu, \nu \in \mathbb{R}, \operatorname{rank}\left(\begin{array}{cc}\beta & \gamma \\ \eta & \theta \\ \lambda & \mu\end{array}\right)=2$. However, in the suitable coordinate system it is possible assume $\alpha=\zeta=\kappa=0$, $\beta=\theta=1, \gamma=\eta=\lambda=\mu=0$. We have

$$
\begin{aligned}
& x=s+\delta s t \\
& y=t+\iota s t \\
& z=\nu s t
\end{aligned}
$$

it follows that

$$
x y-\frac{\delta}{\nu} y z-\frac{\iota}{\nu} x z+\frac{\delta \iota}{\nu^{2}} z^{2}-\frac{1}{\nu} z=0
$$


and by standard procedure we evaluate that it embodies the hyperbolic paraboloid with the normal equation

$$
\begin{array}{r}
\frac{\delta \iota+\sqrt{\left(\delta^{2}+\nu^{2}\right)\left(\iota^{2}+\nu^{2}\right)}}{\nu^{2}} x^{2}+\frac{\delta \iota-\sqrt{\left(\delta^{2}+\nu^{2}\right)\left(\iota^{2}+\nu^{2}\right)}}{\nu^{2}} y^{2} \\
-\frac{2 \nu^{2}}{\sqrt{\delta^{2}+\iota^{2}+\nu^{2}}} z=0 .
\end{array}
$$

Hence $\mathcal{S}_{3}^{*}[\mathbb{D} \otimes \mathbb{D}]=\mathcal{S}_{3}[\mathbb{D} \otimes \mathbb{D}]$ was proved.

\section{Liftings of 1-Forms to $K^{A}$}

3.1. Groundwork. Let $\mathcal{M} f_{m}$ denote the category of $m$-dimensional manifolds (and local diffeomorphisms), $M$ be an object in $\mathcal{M} f_{m}$, $F$ be a natural bundle and $\Omega^{1} M$ be the space of 1 -forms on $M$. Let $\omega \in \Omega^{1} M$. If we denote by $p: F M \rightarrow M$ the natural bundle projection and by $p^{*} \omega$ the pullback $\omega$ to $F$ with respect to $p$, then $p^{*} \omega$ represents a 1-form on $F M$ called the vertical lift of $\omega$ to $F M$. (The stars * denote pullbacks in the rest of the paper.) From now on we assume $A=\mathcal{E}_{k} / \underline{i}, \mathrm{w}(A)=k$ and regard $x^{1}, \ldots, x^{m}$ as usual coordinates on $\mathbb{R}^{m}, \frac{\partial}{\partial x^{1}}, \ldots, \frac{\partial}{\partial x^{m}}$ the canonical vector fields on $\mathbb{R}^{m}, d x^{1}, \ldots, d x^{m}$ the canonical 1 -forms on $\mathbb{R}^{m}$ and $t^{1}, \ldots, t^{k}$ the usual coordinates on $\mathbb{R}^{k}$. We denote by $\pi: \operatorname{reg} T^{A} \mathbb{R}^{m} \rightarrow K^{A} \mathbb{R}^{m}$ the canonical projection. Let $\sigma=\pi\left(j^{A}\left(t^{1}, \ldots, t^{k}, 0, \ldots, 0\right)\right) \in K_{0}^{A} \mathbb{R}^{m}$. We consider a natural operator (regular lifting) $\mathcal{A}: T^{*} \rightsquigarrow T^{*} K^{A}$ and define $\Phi_{\mathcal{A}}: \Omega^{1} \mathbb{R}^{m} \rightarrow \mathbb{R}$ by

$$
\Phi_{\mathcal{A}}:=\left\langle\mathcal{A}(\omega)(\sigma), \mathcal{K}_{\mathbb{R}^{m}}^{A}\left(\frac{\partial}{\partial x^{m}}\right)(\sigma)\right\rangle,
$$

where $\mathcal{K}_{M}^{A}(X)$ is the complete lift of a vector field $X$ to $K^{A} M$, see [9]. Then we have:

Lemma 5. If $\Phi_{\mathcal{A}}=0$, then $\mathcal{A}=0$.

Proof. Since $m \geq \mathrm{w}(A)+1$, the orbit of $\mathcal{K}_{\mathbb{R}^{m}}^{A}\left(\frac{\partial}{\partial x^{m}}\right)(\sigma)$ with respect to diffeomorphisms $\mathbb{R}^{m} \rightarrow \mathbb{R}^{m}$ preserving germ $0\left(\frac{\partial}{\partial x^{m}}\right)$ forms a dense subset in $\left(T K^{A} \mathbb{R}^{m}\right)_{0}$. By the naturality of $\mathcal{A}, \mathcal{A}=0$ follows from $\Phi_{\mathcal{A}}=0$. We use also the regularity of $\mathcal{A}$ and the fact that any nonvanishing vector field is locally $\frac{\partial}{\partial x^{m}}$ in some local coordinates.

This lemma means that $\mathcal{A}$ is uniquely determined by $\Phi_{\mathcal{A}}$ and that is why it is sufficient to study $\Phi_{\mathcal{A}}$ subsequently. Using the naturality of $\mathcal{A}$, we obtain: 
Lemma 6. If $\phi: \mathbb{R}^{m} \rightarrow \mathbb{R}^{m}$ is a diffeomorphism preserving $\mathcal{K}_{\mathbb{R}^{m}}^{A}\left(\frac{\partial}{\partial x^{m}}\right)(\sigma)$, then $\Phi_{\mathcal{A}}(\omega)=\Phi_{\mathcal{A}}\left(\phi^{*} \omega\right)$ for any $\omega \in \Omega^{1} \mathbb{R}^{m}$.

Proof. The lemma is trivial.

Furthermore:

Lemma 7. Let $\omega \in \Omega^{1} \mathbb{R}^{m}$ have the form

$$
\begin{aligned}
\omega=\left(f_{1} \circ\left(x^{1}, \ldots, x^{k}, x^{m}\right)\right) d x^{1} & +\cdots+\left(f_{k} \circ\left(x^{1}, \ldots, x^{k}, x^{m}\right)\right) d x^{k} \\
& +\left(g \circ\left(x^{1}, \ldots, x^{k}, x^{m}\right)\right) d x^{m}, \quad
\end{aligned}
$$

where $f_{1}, \ldots, f_{k}, g: \mathbb{R}^{k+1} \rightarrow \mathbb{R}$ are smooth maps. If $\Phi_{\mathcal{A}}(\omega)=0$ for any such $\omega$, then $\Phi_{\mathcal{A}}=0$.

Proof. Let us assume $\omega \in \Omega^{1} \mathbb{R}^{m}$ be arbitrary. The diffeomorphisms $\phi_{\tau}: \mathbb{R}^{m} \rightarrow \mathbb{R}^{m}$,

$$
\phi_{\tau}:\left(x^{1}, \ldots, x^{m}\right) \mapsto\left(x^{1}, \ldots, x^{k}, \tau x^{k+1}, \ldots, \tau x^{m-1}, x^{m}\right),
$$

$\tau \neq 0$, preserve $\mathcal{K}_{\mathbb{R}^{m}}^{A}\left(\frac{\partial}{\partial x^{m}}\right)(\sigma)$, as they preserve $\frac{\partial}{\partial x^{m}}$ and $\sigma$. We have $\Phi_{\mathcal{A}}(\omega)=\Phi_{\mathcal{A}}\left(\phi_{\tau}^{*} \omega\right)$ by Lemma 6 and for $\tau \rightarrow 0$ we obtain $\Phi_{\mathcal{A}}(\omega)=\Phi_{\mathcal{A}}\left(\phi_{0}^{*} \omega\right)=0$, because $\phi_{0}^{*} \omega$ is of the form (10).

Lemma 8. Let $\tilde{\omega} \in \Omega^{1} \mathbb{R}^{m}$ have a form

$$
\tilde{\omega}=q^{*} \omega_{0}+x^{m} q^{*} \omega_{1}+(H \circ q) d x^{m},
$$

where $\omega_{0}, \omega_{1} \in \Omega^{1} \mathbb{R}^{k}$ are arbitrary, $H: \mathbb{R}^{k} \rightarrow \mathbb{R}$ and $q: \mathbb{R}^{m}=\mathbb{R}^{k} \times$ $\mathbb{R}^{m-k} \rightarrow \mathbb{R}^{k}$ is the projection. If $\Phi_{\mathcal{A}}(\tilde{\omega})=0$ for any such $a \tilde{\omega}$, then $\Phi_{\mathcal{A}}=0$.

Proof. Let us assume that $\omega \in \Omega^{1} \mathbb{R}^{m}$ is of the form (10). As to Lemma 7, it is sufficient to show that $\Phi_{\mathcal{A}}(\omega)=0$. By the corollary of the non-linear Peetre theorem ([6]) for the case of the local operator $\left\langle\mathcal{A}, \mathcal{K}_{\mathbb{R}^{m}}^{A}\left(\frac{\partial}{\partial x^{m}}\right)\right\rangle: \Omega^{1} \mathbb{R}^{m} \rightarrow C^{\infty}\left(K^{A} \mathbb{R}^{m}, \mathbb{R}\right)$, the section $\omega$ and the compact set $\{\sigma\} \subset K^{A} \mathbb{R}^{m}$, there is a $r=r(\omega) \in \mathbb{N}$ such that $\Phi_{\mathcal{A}}(\bar{\omega})=\Phi_{\mathcal{A}}(\omega)$ for any $\bar{\omega} \in \Omega^{1} \mathbb{R}^{m}$ with $j_{0}^{r} \bar{\omega}=j_{0}^{r} \omega$. So, we can assume that $\omega$ is of the form (10) with $f_{1}, \ldots, f_{k}, g: \mathbb{R}^{k+1} \rightarrow \mathbb{R}$ being polynomials of degree at most $r$.

Let us denote $\Phi_{\mathcal{A}}^{r}$ the restriction of $\Phi_{\mathcal{A}}$ to the (finite dimensional) vector space of all forms having the form (10) with $f_{1}, \ldots, f_{k}, g$ being polynomials of degree at most $r$. Since $\mathcal{A}$ satisfies the regularity condition, $\Phi_{\mathcal{A}}^{r}$ is smooth. The diffeomorphisms $\eta_{\tau}: \mathbb{R}^{m} \rightarrow \mathbb{R}^{m}$, 
$\eta_{\tau}:\left(x^{1}, \ldots, x^{m}\right) \mapsto\left(x^{1}, \ldots, x^{m-1}, \tau x^{m}\right), \tau \neq 0$, preserve $\sigma$ and they send $\frac{\partial}{\partial x^{m}}$ to $\tau \frac{\partial}{\partial x^{m}}$. Similarly as for Lemma 6 , we deduce

$$
\begin{aligned}
\tau \Phi_{\mathcal{A}}^{r}(\omega)=\Phi_{\mathcal{A}}^{r}\left(\eta_{\tau}^{*} \omega\right)= & \\
\Phi_{\mathcal{A}}^{r}\left(\left(f_{1} \circ\left(x^{1}, \ldots, x^{k}, \tau x^{m}\right)\right) d x^{1}\right. & +\cdots+\left(f_{k} \circ\left(x^{1}, \ldots, x^{k}, \tau x^{m}\right)\right) d x^{k} \\
& \left.+\tau\left(g \circ\left(x^{1}, \ldots, x^{k}, \tau x^{m}\right)\right) d x^{m}\right) .
\end{aligned}
$$

Differentiating both sides of this formula and of the similar formula with $\tilde{\omega}=\left(f_{1} \circ\left(x^{1}, \ldots, x^{k}, 0\right)+\frac{\partial f_{1}}{\partial x^{m}} \circ\left(x^{1}, \ldots, x^{k}, 0\right) x^{m}\right) d x^{1}+\cdots+\left(f_{k} \circ\right.$ $\left.\left(x^{1}, \ldots, x^{k}, 0\right)+\frac{\partial f_{k}}{\partial x^{m}} \circ\left(x^{1}, \ldots, x^{k}, 0\right) x^{m}\right) d x^{k}+\left(g \circ\left(x^{1}, \ldots, x^{k}, 0\right)\right) d x^{m}$ instead of $\omega$ with respect to $\tau$ and then putting $\tau=0$, we derive $\Phi_{\mathcal{A}}^{r}(\omega)=\Phi_{\mathcal{A}}^{r}(\tilde{\omega})$. Nevertheless, $\tilde{\omega}$ is of the form (11). Then using the assumption of the lemma we obtain $\Phi_{\mathcal{A}}(\omega)=0$.

Lemma 9. If $\Phi_{\mathcal{A}}\left(\alpha d x^{m}\right)=0$ for any $\alpha \in \mathbb{R}$, then $\Phi_{\mathcal{A}}=0$.

Proof. Let $\tilde{\omega} \in \Omega^{1} \mathbb{R}^{m}$ be of the form (11). Let us choose a sequence $\left\{\phi_{n}\right\}_{n=1}^{\infty}$ of automorphisms $\phi_{n} \in$ Aut $A$ such that $\phi_{n} \rightarrow \kappa_{A}$ for $n \rightarrow \infty$. Then there exists a sequence $\left\{\Phi_{n}\right\}_{n=1}^{\infty}$ of diffeomorphisms $\Phi_{n}: \mathbb{R}^{k} \rightarrow \mathbb{R}^{k}, \Phi_{n}(0)=0$, with $j_{0}^{\infty} \Phi_{n} \rightarrow 0$ such that $\Phi_{n}^{*}(\underline{\mathfrak{i}})=\underline{\mathfrak{i}}$ and $\phi_{n}=\left[\Phi_{n}^{*}\right]$ is the quotient homomorphism. We define $\Psi_{n}: \mathbb{R}^{m} \rightarrow$ $\mathbb{R}^{m}, \Psi_{n}:=\Phi_{n} \times \operatorname{id}_{\mathbb{R}^{m-k}}$. Then $\Psi_{n}$ preserve $\mathcal{K}_{\mathbb{R}^{m}}^{A}\left(\frac{\partial}{\partial x^{m}}\right)(\sigma)$, as they preserve $\frac{\partial}{\partial x^{m}}$ (because $m \geq k+1$ ) and $\sigma$ (because $K^{A} \Psi_{n}(\sigma)=$ $\pi\left(j^{A} \Psi_{n}\left(t^{1}, \ldots, t^{k}, 0, \ldots, 0\right)\right)=\pi\left(j^{A}\left(t^{1}, \ldots, t^{k}, 0, \ldots, 0\right) \circ \Phi_{n}\right)=$ $\left.\pi\left(\phi_{n}\left(j^{A}\left(t^{1}, \ldots, t^{k}, 0, \ldots, 0\right)\right)\right)=\pi\left(j^{A}\left(t^{1}, \ldots, t^{k}, 0, \ldots, 0\right)\right)=\sigma\right)$.

According to Lemma 6,

$\Phi_{\mathcal{A}}(\tilde{\omega})=\Phi_{\mathcal{A}}\left(\Psi_{n}^{*} \tilde{\omega}\right)=\Phi_{\mathcal{A}}\left(q^{*}\left(\Phi_{n}^{*} \omega_{0}\right)+q^{*}\left(\Phi_{n}^{*} \omega_{1}\right) x^{m}+\left(H \circ \Phi_{n} \circ q\right) d x^{m}\right)$.

But $j_{0}^{\infty}\left(\Phi_{n}^{*} \omega_{0}\right) \rightarrow 0, j_{0}^{\infty}\left(\Phi_{n}^{*} \omega_{1}\right) \rightarrow 0$ and $j_{0}^{\infty}\left(H \circ \Phi_{n}\right) \rightarrow j_{0}^{\infty}(H(0))$ as $n \rightarrow \infty$. Then $\Phi_{\mathcal{A}}(\tilde{\omega})=\Phi_{\mathcal{A}}\left(H(0) d x^{m}\right)=0$ because of the assumption. Next we use Lemma 8.

3.2. The classification theorem. Thanks to the thorough groundwork, we are now in position to prove the following main theorem without effort.

Theorem 1. Let $A$ be a dwindlable Weil algebra, $m \geq \mathrm{w}(A)+1$. Then every natural operator $\mathcal{A}: T^{*} \rightsquigarrow T^{*} K^{A}$ is a constant multiple of the vertical lifting. 
Proof. The operator $\mathcal{A}$ is determined uniquely by

$$
\Psi_{\mathcal{A}}: \mathbb{R} \rightarrow \mathbb{R}, \quad \Psi_{\mathcal{A}}(\alpha):=\Phi_{\mathcal{A}}\left(\alpha d x^{m}\right)
$$

according to Lemma 9. If we take into account the homotheties

$$
\left(x^{1}, \ldots, x^{m}\right) \mapsto\left(x^{1}, \ldots, x^{m-1}, \tau x^{m}\right),
$$

we deduce by the naturality of $\mathcal{A}$ that $\Psi_{\mathcal{A}}(\tau \alpha)=\tau \Psi_{\mathcal{A}}(\alpha)$, i.e. $\Psi_{\mathcal{A}}$ is linear as to the homogeneous function theorem, see e.g. [6]. Hence $\mathcal{A}$ is determined uniquely by the real number $\Psi_{\mathcal{A}}(1)$. Therefore the vector space of all natural operators $\mathcal{A}: T^{*} \rightsquigarrow T^{*} K^{A}$ has dimension at most 1 . The proof is complete.

Corollary. If $A=\mathbb{D}_{k_{1}}^{r_{1}} \otimes \cdots \otimes \mathbb{D}_{k_{l}}^{r_{l}}, m \geq \mathrm{w}(A)+1$. Then every natural operator $\mathcal{A}: T^{*} \rightsquigarrow T^{*} K^{A}$ is a constant multiple of the vertical lifting.

Proof. Every Weil algebra $A=\mathbb{R}\left[t^{1}, \ldots, t^{k}\right] / \mathfrak{i}$, where $\mathfrak{i}$ is a homogeneous ideal, is dwindlable.

REMARK 4. Up to now, only the special case of the Corollary for $l=1$ was known, see [7].

\section{REFERENCES}

1. R. J. Alonso, Jet manifolds associated to a Weil bundle, Arch. Math. (Brno) 36 (2000), 195-199.

2. C. Ehresmann, Introduction á la théorie des structures infinitésimales et des pseudo-groupes de Lie, in: Colloque du C.N.R.S., Strasbourg (1953), 97-110.

3. J. Gancarzewicz, W. Mikulski, Z. Pogoda, Natural bundles and natural liftings. Prolongation of geometric structures, in: Proc. Conf. on Diff. Geom. and Its Appl., Opava 1992, Silesian University Opava (1993), 281-320.

4. H. Guggenheimer, Contact elements, contact correspondences and contact invariants, Ann. Mat. Pura Appl., IV. Ser. 120 (1979), 229-261.

5. F. Guil-Asenssio, M. Saorín, The group of automorphisms of a commutative algebra, Math. Z. 219 (1995), 31-48.

6. I. Koláŕ, P. W. Michor, J. Slovák, Natural Operations in Differential Geometry, Springer-Verlag 1993.

7. I. Kolář, W. Mikulski, Contact elements on fibered manifolds, Czech. Math. J., to appear.

8. M. Kureš, Weil algebras of generalized higher order velocities bundles, in: Global differential geometry: the mathematical legacy of Alfred Gray (Bilbao, 2000), Amer. Math. Soc., Contemp. Math., 288 (2001), 358-362.

9. M. Kureš, W. Mikulski, Natural operators lifting vector fields to bundles of Weil contact elements, Czech. Math. J., to appear.

10. W. Mikulski, The natural operators lifting 1-forms to the bundles of $A$ velocities, Mh. Math. 119 (1995) 63-77. 
Miroslav Kureš,

Department of Mathematics,

Brno University of Technology,

Technická 2, 61669 Brno, Czech Re-

public

kures@mat.fme.vutbr.cz
Włodzimierz M. Mikulski,

Institute of Mathematics,

Jagiellonian University,

Reymonta 4, 30-059 Kraków,

Poland

mikulski@im.uj.edu.pl

Received on 25 October 2001. 